\title{
Clinical Problem Solving and Using New Paths in the Laboratory: Learning from Case Studies*
}

\author{
Bernhard Lämmle ${ }^{1,2,3}$
}

\footnotetext{
${ }^{1}$ Department of Hematology and Central Hematology Laboratory, Inselspital, Bern University Hospital, University of Bern, Bern, Switzerland

${ }^{2}$ Center for Thrombosis and Hemostasis, University Medical Center, Johannes Gutenberg University, Mainz, Germany

${ }^{3}$ Haemostasis Research Unit, University College London, London,

United Kingdom
}

Hämostaseologie 2020;40:414-419.
Address for correspondence Bernhard Lämmle, Emeritus Professor, MD, Schützenweg 3, CH 3065 Bolligen, Bern, Switzerland (e-mail: bernhard.laemmle@uni-mainz.de).

\author{
Abstract \\ Keywords \\ - bleeding \\ complications \\ - vitamin K antagonist \\ - F9 propeptide \\ mutation \\ - congenital \\ - autoimmune \\ - thrombotic \\ thrombocytopenic \\ purpura \\ - ADAMTS13 \\ - patent ductus \\ arteriosus \\ - case reports
}

Three selected casuistic studies of individual patient problems from my long-standing professional experience are presented. I intend to highlight the potential contribution of case studies, including new approaches of laboratory investigation, for the advancement of pathophysiological knowledge and would encourage to "rehabilitate" the low academic profile generally attributed to "case reports."

\section{Introduction}

Case reports are generally considered to be of low value neither contributing to solid medical knowledge nor promoting scientific progress. Medical faculties may not attribute academic credit for case reports, e.g., for promotion to clinician-scientist or habilitation (e.g., Informationen zur Habilitation der Universitätsmedizin Mainz: http://www. um-mainz.de/rfl/wissenschaftliche-nachwuchsfoerderung/ habilitationen/informationen-zur-habilitation.html).

Nevertheless, I believe that case studies, reflecting the daily approach of physicians confronted with complex clinical problems, can well serve as starting points for relevant

\footnotetext{
* Presented as a lecture at the 64th Annual Meeting of the GTH in Bremen, February 18-21, 2020.
}

received

May 11, 2020

accepted after revision

June 29, 2020 translational research. Here, I will present three case reports from my 45-year-long clinical activity and show that studying patients presenting with unclear medical conditions and applying new laboratory investigations may result in an improved understanding of disease mechanisms and ultimately better treatment.

\section{Case 1}

In June 1997, a male patient born in 1939 was referred to the outpatient ward of the University Clinic of Hematology at Inselspital, Bern, for investigation of the cause of recurrent bleeding on phenprocoumon (Marcoumar ${ }^{\circledR}$ ) treatment. He had undergone tonsillectomy as a child and varicose vein stripping on both legs at the age of 37 years without bleeding complications. Diabetes mellitus type 2 , hyperuricemia, and
DOI https://doi.org/ 10.1055/a-1191-7198. ISSN 0720-9355.
(C) 2020 Georg Thieme Verlag KG Stuttgart · New York 
arterial hypertension were well controlled. In January 1996 , a mechanical aortic valve prosthesis (St. Jude Medical) was inserted without any perioperative complication and he started vitamin $\mathrm{K}$ antagonist treatment with phenprocoumon targeting at an international normalized ratio (INR) of 2 to 3. In April 1996 he suffered an intramuscular hematoma in the left quadriceps muscle, 1 month later an intra-articular bleed in his left knee joint, and in August macrohematuria occurred. In November 1996, an acute gastrointestinal bleeding with intramural hematoma, ileus, and resulting anemia necessitated hospitalization. After recovery, he suffered from muscle hematoma in his right leg in January 1997, bleeding on the right arm in March 1997, and shortly thereafter a lifethreatening pharyngeal-laryngeal bleed. Of note, all these recurring hemorrhagic complications occurred at INR values within the therapeutic range.

While bleeding is the most common side effect of anticoagulant treatment, this series of severe bleeding complications at therapeutic INR values suggested an additional underlying hemostatic defect despite the fact that the patient had never shown any abnormal hemorrhagic tendency until his heart valve prosthesis placement.

Hemostasis testing in June 1997 showed normal platelet aggregation studies in platelet-rich plasma using various agonists, and von Willebrand factor (VWF) activity and antigen, $\alpha 2$-antiplasmin, and factor XIII activity were normal. Prothrombin time measured as Quick percentage was 54\%; INR 1.5 while on phenprocoumon. The activated partial thromboplastin time (aPTT) was 77.3 seconds (normal: 25-36 seconds), factor [F] II:C 56\%, FVII:C 78\%, FX:C 27\%, and FIX:C 3\%.

The disproportionately prolonged aPTT at an INR of 1.5 under vitamin $\mathrm{K}$ antagonist therapy was due to a severely decreased FIX: $\mathrm{C}$ and this phenomenon of a FIX sensitivity to vitamin $\mathrm{K}$ antagonist therapy had just been reported in an individual Caucasian patient living in the United States by Chu et $\mathrm{al}^{1}$ and in three German patients by Oldenburg et $\mathrm{al}^{2} \mathrm{a}$ few months before our Swiss patient was evaluated in Bern. Similarly to our patient these four reported cases showed severe bleeding complications while on vitamin $\mathrm{K}$ antagonist therapy, whereas no hemorrhagic diathesis was evident in the absence of coumarins.

Genomic DNA of our patient was analyzed by Prof. Oldenburg and an F9 (NM_000133.4): c.110C > T, p.Ala37Val (old terminology Ala-10 $\rightarrow$ Val) mutation in the FIX propeptide was established.

\section{Comment to Case 1}

As illustrated in - Fig. 1, vitamin K-dependent coagulation factors are synthesized as inactive zymogens in the liver and are then gamma-carboxylated at several glutamic acid residues to become active, i.e., being able to bind $\mathrm{Ca}^{2+}$ ions and thereby adopting a conformation allowing to bind to negatively charged phospholipid surfaces. ${ }^{3}$ The $\gamma$-glutamyl carboxylase with vitamin $\mathrm{K}$ hydroquinone as cofactor binds to the carboxylase recognition site located on the propeptide region of the zymogen. Chu et al studied the observed phenomenon of coumarin sensitivity of FIX in their unique patient by in vitro $\gamma$-carboxylation studies of FIX peptides containing either the wild-type or mutant FIX propeptide sequences. ${ }^{1}$ They showed that the $K_{\mathrm{m}}$ values for carboxylation of the p.Ala37Thr (old terminology Ala-10 $\rightarrow$ Thr) variant was 33-fold higher than that for the wild-type FIX peptide, whereas the $V_{\text {max }}$ values differed only minimally. ${ }^{1}$ Thus, the p.Ala37Thr propeptide variant has a much lower binding affinity for the gamma-glutamyl carboxylase, obviously allowing a sufficient gamma-carboxylation of FIX in the presence of normal amounts of vitamin $\mathrm{K}$ but not in the presence of vitamin $\mathrm{K}$ antagonists.

Shortly after Chu et al's report on a single patient in October 1996, ${ }^{1}$ three similar patients were observed in Germany, displaying either F9 (NM_000133.4): c.110C > T, p. Ala37Val (old terminology Ala-10 $\rightarrow$ Val), or c.109G $>$ A, p. Ala37Thr (old terminology Ala- $10 \rightarrow$ Thr). ${ }^{2}$ The fact that briefly thereafter the patient presented here as Case 1 was discovered triggered awareness of this important and highly dangerous condition. Several additional cases were detected in the following years, and by 2001 Oldenburg et al compiled all 11 cases with the same clinical phenotype of severe bleeding complications under vitamin $\mathrm{K}$ antagonist treatment but with normal hemostasis in its absence. ${ }^{4}$ Haplotype analysis of the $F 9$ gene suggested two distinct founder mutations in the five German and Swiss patients with the p.Ala37Val variant as well as in four individuals from Germany, Switzerland, and Great Britain with the p.Ala37Thr variant, respectively, whereas two p.Ala37Thr variants discovered in patients from the United States showed independent de novo mutations. ${ }^{4}$ These $F 9$ propeptide mutations are very rare ${ }^{5}$ and do not justify general screening before coumarin therapy. Nevertheless, unexpected bleeding at therapeutic INR values mandates assay of the aPTT followed by FIX: $\mathrm{C}$ measurement in case of disproportionate aPTT prolongation. The careful study of these rare patients with a highly pathologic bleeding phenotype while on therapeutic dose vitamin $\mathrm{K}$ antagonist treatment has unraveled a new type of clinically relevant coumarin sensitivity which up to 1996 had escaped detection in all large trials on vitamin $\mathrm{K}$ antagonists.

\section{Case 2}

A man born in 1964 was hospitalized in 1985 because of vomiting, abdominal discomfort, icterus, somnolence, and petechial bleeding on both feet. Hemoglobin was $10.8 \mathrm{~g} / \mathrm{dL}$, platelet count was $15 \times 10^{9} / \mathrm{L}$, plasma hemoglobin was $0.035 \mathrm{~g} / \mathrm{L}$ (normal $<0.01$ ), and s-creatinine was $846 \mu \mathrm{mol} / \mathrm{L}$ (normal $<116$ ). Hemolytic uremic syndrome was diagnosed and the patient received hemodialysis. Seven months later he had an episode with fever and macrohematuria after aspirin intake, and screatinine had almost normalized (120 $\mu \mathrm{mol} / \mathrm{L})$. Recurring episodes of hemolysis and thrombocytopenia occurred between 1987 and 1989 when he suffered from hematuria and renal failure prompting transient hemodialysis. This was shortly followed by an acute ischemic stroke at a time of severe thrombocytopenia. During 1990 to 1991, four acute bouts of thrombocytopenia and hemolysis were controlled by plasma exchange and fresh frozen plasma (FFP) replacement. In 1992 prophylactic plasma exchange with FFP replacement was introduced and performed every 4 months. In 1996 the patient was 


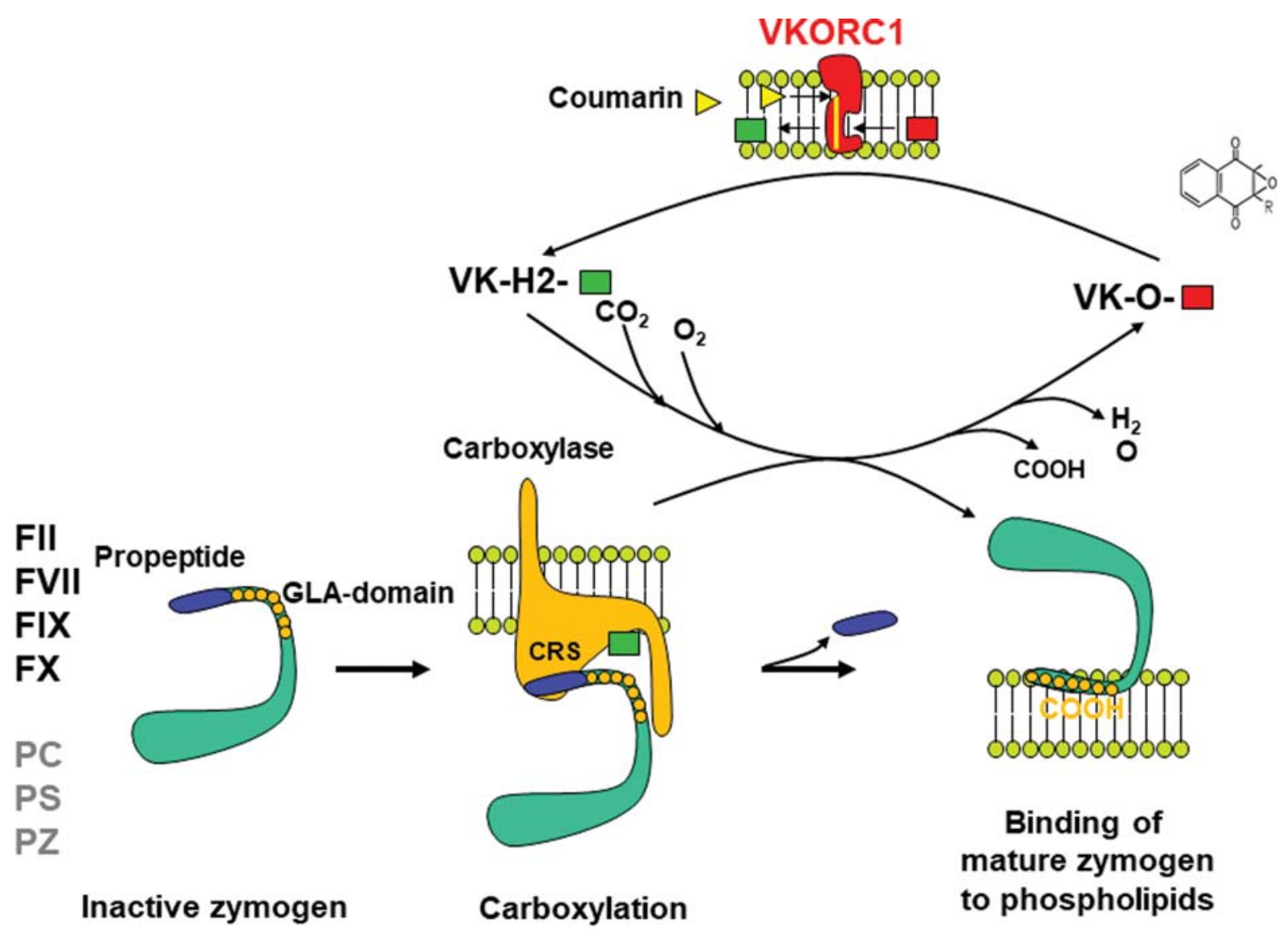

\section{J. Oldenburg. Hämostaseologie 2006;26:13-21}

Fig. 1 Gamma-carboxylation of vitamin K-dependent coagulation factors. The $\mathrm{Y}$-glutamyl carboxylase, with vitamin K hydroquinone (VK-H2) as cofactor, binds to the carboxylase recognition site (CRS) located on the propeptide of the vitamin K-dependent zymogens and introduces $\gamma$ carboxyl groups $(\mathrm{COOH})$ to several glutamic acid residues in the Gla domain resulting in functionally active zymogens that are able to bind $\mathrm{Ca}^{2+}$ and attach to negatively charged phospholipid surfaces. During this carboxylation reaction vitamin Kepoxide (VK-O) is generated that is reduced in two steps to vitamin K quinone (not shown) and vitamin K hydroquinone (VK-H2) by vitamin K epoxide reductase C1 (VKORC1). Coumarin antagonizes VK-O reduction by VKORC1. (Modified with permission from Oldenburg 2006 ${ }^{3}$.)

diagnosed with invalidating psychoorganic syndrome, platelets were most often at a subnormal level, and lactate dehydrogenase (LDH) was constantly elevated. Magnetic resonance imaging of the brain unraveled multiple residues of ischemic infarctions and right temporal lobe atrophy.

The patient's brother, born in 1975, had been hospitalized at the age of 2 years because of severe hemolysis. Recurrent bouts of hemolysis and thrombocytopenia seemed to respond promptly to plasma exchange and, therefore, as in his brother, prophylactic plasma exchange and FFP replacement were started in the mid-1990s.

These two brothers were reminiscent of the patient reported by Upshaw in $1978 .{ }^{6}$ This young woman suffered from recurrent thrombocytopenia and microangiopathic hemolytic anemia and repeatedly showed prompt responses to infusion of plasma ( - Fig. 2).

In 1982, Moake et al reported four patients suffering from chronic relapsing thrombotic thrombocytopenic purpura (TTP) displaying unusually large VWF (ULVWF) multimers, presumed to be causally linked to the recurring platelet clumping in the microcirculation during acute TTP attacks. ${ }^{7}$ In 1996,
Furlan and coworkers in Bern and Tsai in New York independently discovered a so far unknown metalloprotease isolated from human plasma that was able to cleave VWF at the peptide bond Tyr842-Met843 (Tyr1605-Met1606 including the propeptide sequence in the amino acid numbering $)^{8,9}$ ( - Fig. 3 ).

Testing the plasma samples of the two brothers showed a completely deficient activity of this VWF-cleaving protease explaining the presence of ULVWF multimers in their plasma. Father and mother had approximately $50 \%$ of VWF-cleaving protease activity ( - Fig. 4 A, B) ${ }^{10}$ These two brothers with an obviously hereditary form of recurrent TTP represented the first patients where a severe deficiency of the specific VWFcleaving protease, nowadays denoted as A Disintegrin And Metalloprotease with ThromboSpondin type 1 domains, number 13 (ADAMTS13) ${ }^{12,13}$ was uncovered.

\section{Comment to Case 2}

The serendipitous testing of the two brothers suffering from life-long recurrent TTP attacks for the newly discovered VWF-cleaving protease activity finally shed light on the pathophysiology of this severe and often fatal disease, 


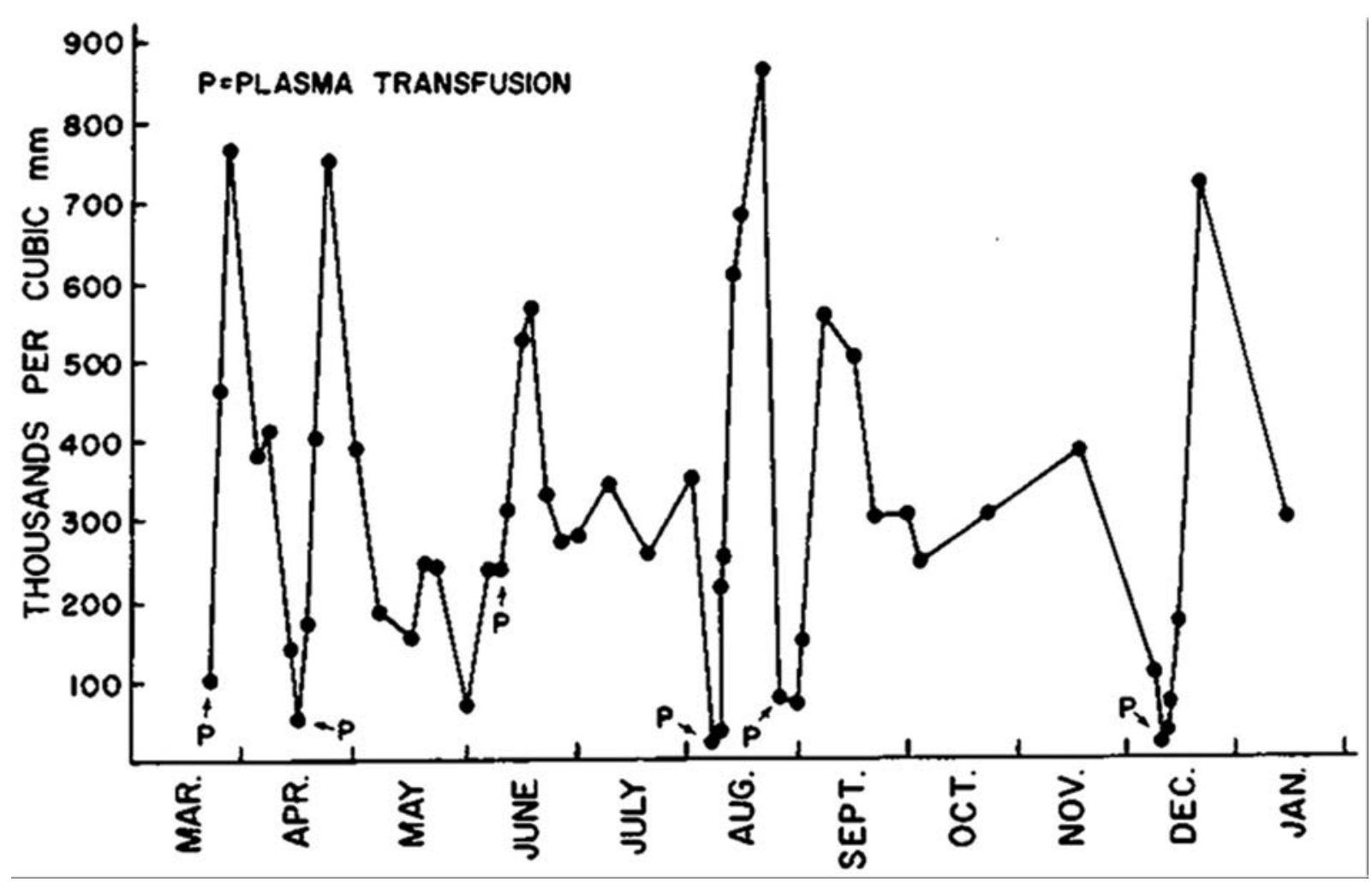

Fig. 2 Platelet count responses to fresh frozen plasma infusions $(\mathrm{P})$ over a period of 10 months in a patient with recurring microangiopathic hemolysis and thrombocytopenia. (Adapted with permission from Upshaw 19786.)

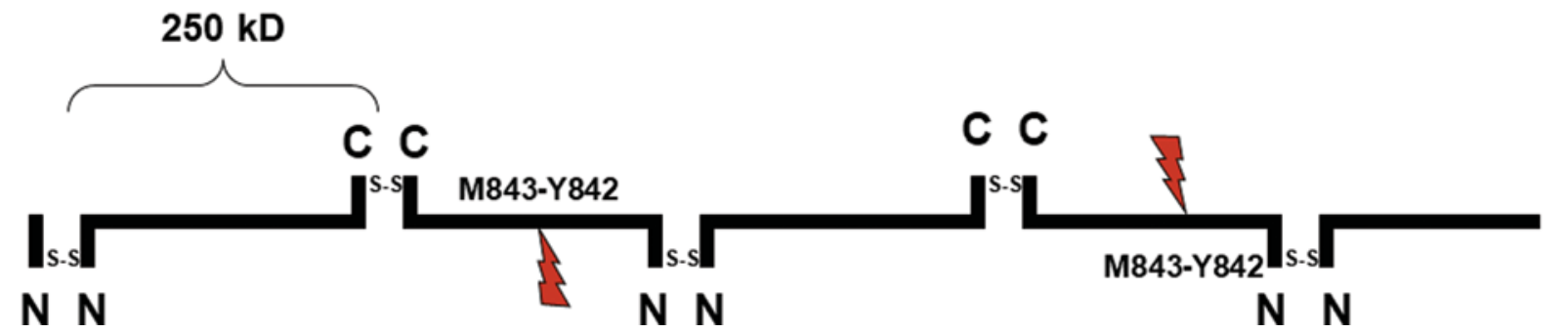

Fig. 3 Schematic presentation of proteolytic cleavage of von Willebrand factor (VWF) subunits by the specific VWF-cleaving protease (symbolized by jagged arrow), initially described in $1996^{8,9}$ and now denoted as ADAMTS13. Cleavage at tyrosine (Y) 842-methionine (M) 843 (nowadays denoted Y1605-M1606, including the propeptide sequence in amino acid numbering). (Prof. J. A. Kremer Hovinga is acknowledged for preparing this figure).

initially described by Moschcowitz in $1924 .{ }^{14}$ The case study outlined above demonstrates the potential importance of translational research starting from the bedside observation of a major clinical problem, chronic relapsing TTP, to the bench where basic research on the proteolytic processing of VWF had resulted in the discovery of a new enzyme and the fortuitous finding that a severe deficiency of the latter was the main pathophysiological cause of the disease.

During the past 25 years, many researchers and clinicians have broadly extended the knowledge on the pathogenesis, diagnosis and differential diagnosis, clinical manifestations, treatment, and long-term outcome of both congenital TTP (cTTP) and the still rare but more common acquired autoimmune TTP (iTTP) where autoantibodies inactivating and/or clearing the ADAMTS13 lead to the clinical manifestations of acute TTP. ${ }^{15-25}$ Of special note, treatment of patients with cTTP may be facilitated in the near future and long-term sequelae diminished by the availability of recombinant human ADAMTS13 that has been tested in a pilot study. ${ }^{19}$ Patients with iTTP may benefit from newly developed treatment strategies. The application, in parallel to plasma exchange and immunosuppressive therapy, of the humanized monoclonal antibody caplacizumab, inhibiting the VWF A1-domain interaction with the platelet glycoprotein $\mathrm{Ib}$ receptor, during the acute disease episode results in faster platelet recovery, less TTP exacerbations and recurrences, less refractoriness to treatment, and faster normalization of disease biomarkers. ${ }^{21}$ This will hopefully translate into less persisting ischemic organ damage. Moreover, the efficacy of caplacizumab proves in retrospect the primary pathogenetic role of the ULVWF-induced platelet consumption initially deduced and postulated based on casuistic observations. ${ }^{7,10}$ 

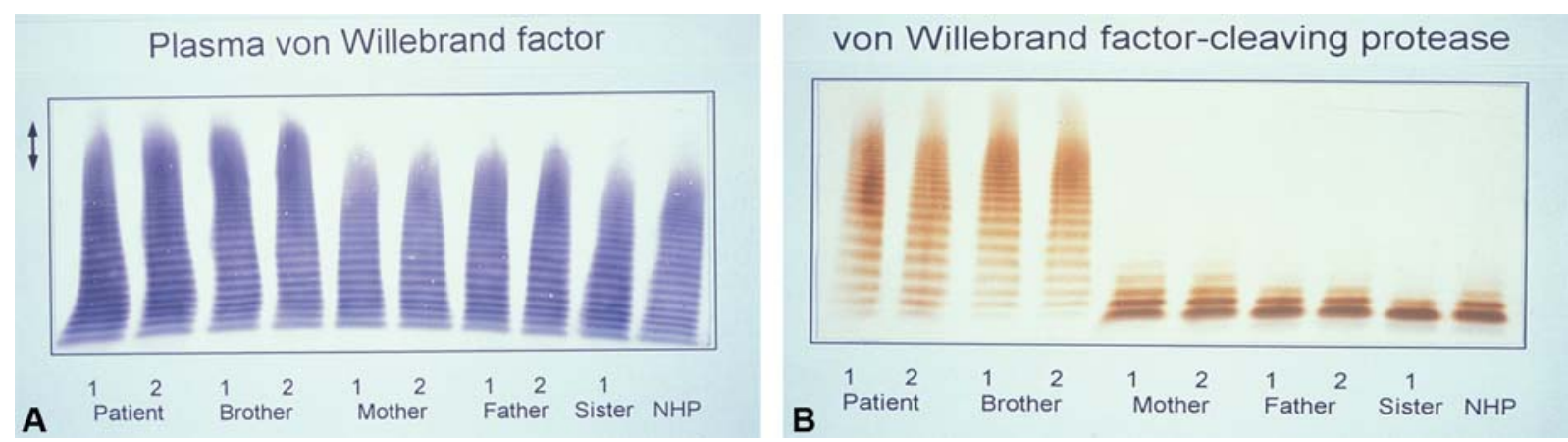

Fig. 4 (A, B) Plasma von Willebrand factor (VWF) multimer analysis (A) and VWF-cleaving protease assay (B) in the index patient, his affected brother, family members, and pooled normal human plasma (NHP) (cases reported in Furlan et al ${ }^{10}$; figure reproduced from Lämmle and von Auer ${ }^{11}$ with permission). (A) VWF multimer analysis on SDS-agarose gel electrophoresis followed by immunoblotting in the two brothers with chronic relapsing TTP (denoted as patient and brother, respectively), mother, father, sister, and pooled NHP. 1 and 2 denote two plasma samples each, obtained several weeks apart without preceding plasma infusion. The two affected brothers showed the presence of unusually large VWF multimers (double arrow). (B) Purified VWF substrate was incubated with diluted plasma samples that had been preactivated by barium chloride from subjects as in - Fig. 4A. The VWF substrate-diluted plasma mixtures were dialyzed overnight against low ionic strength buffer containing $1.5 \mathrm{M}$ urea and then subjected to SDS-agarose electrophoresis and immunoblotting. Both plasma samples of the affected brothers completely lacked VWF-cleaving protease activity, mother's and father's plasma had mildly reduced activity (barely visible for father), whereas sister's plasma had normal activity.

\section{Case 3}

In Japan a large number of patients have been diagnosed with cTTP, also denoted as Upshaw-Schulman syndrome (USS) and caused by biallelic mutations of the ADAMTS13 gene in the past 20 years. ${ }^{26}$ About 25 to $40 \%$ of cTTP patients in the Registry at Nara Medical University presented with a postnatal acute bout of hemolytic jaundice and thrombocytopenia necessitating exchange blood transfusion. This hallmark of USS is distinct from the more common neonatal alloimmune hemolysis, mostly due to transplacental transfer of maternal anti-Rhesus D antibodies, which starts already in utero.

A retrospective review of the 22 USS patients with a documented neonatal hemolytic jaundice and thrombocytopenia undergoing exchange blood transfusion within the first days of life revealed one single hemolytic bout in 21 of the 22 patients. $^{27}$ The remaining patient suffered from three consecutive distinct episodes on postnatal days 1, 12, and 28. After four exchange blood transfusions within the first 2 days of life resulting in a decrease of $\mathrm{LDH}$ and bilirubin and an increase of the platelet count, a new bout of hemolytic jaundice and thrombocytopenia occurred on day 12 after a persisting patent ductus arteriosus Botalli (PDA) had been diagnosed on day 8 . Indomethacin, given with the intent to close the PDA, failed, and after a third bout of hemolysis and platelet count decrease on day 26, surgical closure of the PDA was performed and led to lasting remission until the age of 14 months. ${ }^{27}$

\section{Comment to Case 3}

The observation of three distinct bouts of neonatal hemolytic jaundice in this exceptional cTTP patient had initially been overlooked. ${ }^{28}$ Nevertheless, the retrospective analysis comparing all meticulously documented 22 cTTP patients in the Nara Registry led to the hypothesis that the neonatal hemolytic jaundice and thrombocytopenia seen in many cases of USS may be triggered by the open ductus arteriosus which usually obliterates within the first 48 hours of life when postnatal blood flow reversal resulting in left-to-right shunt of highly oxygenated blood leads to vasoconstriction and closure of the PDA. The high shear rate after postnatal blood flow through the narrow conduit of the PDA may trigger the initial cTTP episode by stretching the ULVWF multimers, hyperadhesive in the absence of any ADAMTS13, that will induce platelet adhesion and aggregation. The single patient in the registry with persisting PDA and recurring neonatal TTP bouts strongly argues in favor of this hypothesis. ${ }^{27}$

\section{Conclusion}

These three casuistic observations demonstrate, in the author's opinion, the potential value of the careful analysis of and research based on individual patient problems. As treating physicians we need to precisely define the clinical problem based on a synoptic evaluation of the patient's history, symptoms, signs, and laboratory results. Applying problem-oriented focused research strategies including, if needed, innovative laboratory techniques, may ultimately provide relevant insights into the pathophysiology of orphan diseases or conditions. The recognition of a rare cause of coumarin sensitivity due to $F 9$ propeptide mutation in a few patients revealed a new mechanism leading to severe bleeding despite well-controlled vitamin $\mathrm{K}$ antagonist treatment. The discovery of ADAMTS13 as the enzyme specifically regulating the size and thereby the adhesiveness of VWF and of its congenital or acquired deficiency as the main pathophysiologic cause of the diseases cTTP and iTTP, respectively, were of utmost importance for the understanding of disease mechanisms and development of new therapeutic strategies. Finally, the precise documentation of patients with cTTP in a registry permitted to put forward the hypothesis that the neonatal hemolytic jaundice and thrombocytopenia in USS neonates are triggered by the high shear stress leading to unfolding of ULVWF, unprocessed in the absence of ADAMTS13, when postnatal blood flow reversal drives 
oxygenated blood through the initially open narrow ductus arteriosus Botalli.

Besides adhering to and aiming for evidence-based medicine by focusing on results of and performing new randomized controlled trials, there is, in my opinion, a clear need for research and use of innovative laboratory approaches starting from individual case studies.

\section{Conflicts of Interest}

B.L. is chairman of the Data Safety Monitoring Committees for the Shire 281102 study (rADAMTS13 in cTTP) and for the Shire SHP 655-201 study (rADAMTS13 in iTTP) (now both sponsored by Takeda); over the past 6 years, B.L. received congress travel support by Baxter, Ablynx, Alexion, Siemens, and Bayer, and lecture fees from Siemens, Bayer, Alexion, Roche, and Sanofi.

\section{Acknowledgment}

I thank all the many colleagues with whom I had and still have the opportunity and pleasure to collaborate for more than 45 years in both clinical management and biomedical research. A special tribute goes to my collaborator and friend, Prof. Miha Furlan, who died in December 2018 and who was a genius with great vision and a long-term friend.

\section{References}

1 Chu K, Wu SM, Stanley T, Stafford DW, High KA. A mutation in the propeptide of factor IX leads to warfarin sensitivity by a novel mechanism. J Clin Invest 1996;98(07):1619-1625

2 Oldenburg J, Quenzel EM, Harbrecht U, et al. Missense mutations at ALA-10 in the factor IX propeptide: an insignificant variant in normal life but a decisive cause of bleeding during oral anticoagulant therapy. Br J Haematol 1997;98(01):240-244

3 Oldenburg J. Current and future prospects in haemostasis [in German]. Hamostaseologie 2006;26(01):13-21

4 Oldenburg J, Kriz K, Wuillemin WA, et al; Study Group on Hereditary Warfarin Sensitivity. Genetic predisposition to bleeding during oral anticoagulant therapy: evidence for common founder mutations (FIXVal-10 and FIXThr-10) and an independent CpG hotspot mutation (FIXThr-10). Thromb Haemost 2001; 85(03):454-457

5 Peters J, Luddington R, Brown K, Baglin C, Baglin T. Should patients starting anticoagulant therapy be screened for missense mutations at Ala-10 in the factor IX propeptide? Br J Haematol 1997;99 (02):467-468

6 Upshaw JD Jr. Congenital deficiency of a factor in normal plasma that reverses microangiopathic hemolysis and thrombocytopenia. N Engl J Med 1978;298(24):1350-1352

7 Moake JL, Rudy CK, Troll JH, et al. Unusually large plasma factor VIII:von Willebrand factor multimers in chronic relapsing thrombotic thrombocytopenic purpura. N Engl J Med 1982;307(23): 1432-1435

8 Furlan M, Robles R, Lämmle B. Partial purification and characterization of a protease from human plasma cleaving von Willebrand factor to fragments produced by in vivo proteolysis. Blood 1996; 87(10):4223-4234

9 Tsai HM. Physiologic cleavage of von Willebrand factor by a plasma protease is dependent on its conformation and requires calcium ion. Blood 1996;87(10):4235-4244
10 Furlan M, Robles R, Solenthaler M, Wassmer M, Sandoz P, Lämmle B. Deficient activity of von Willebrand factor-cleaving protease in chronic relapsing thrombotic thrombocytopenic purpura. Blood 1997;89(09):3097-3103

11 Lämmle B, von Auer C. History of thrombotic thrombocytopenic purpura and the von Willebrand factor-cleaving protease, ADAMTS13. In: GM Rodgers, ed. ADAMTS13, Biology and Disease. Cham: Springer International Publishing; 2015:1-19

12 Levy GG, Nichols WC, Lian EC, et al. Mutations in a member of the ADAMTS gene family cause thrombotic thrombocytopenic purpura. Nature 2001;413(6855):488-494

13 Zheng X, Chung D, Takayama TK, Majerus EM, Sadler JE, Fujikawa K. Structure of von Willebrand factor-cleaving protease (ADAMTS13), a metalloprotease involved in thrombotic thrombocytopenic purpura. J Biol Chem 2001;276(44): 41059-41063

14 Moschcowitz E. Hyaline thrombosis of the terminal arterioles and capillaries: a hitherto undescribed disease. Proc NY Pathol Soc 1924;24:21-24

15 Mariotte E, Azoulay E, Galicier L, et al; French Reference Center for Thrombotic Microangiopathies. Epidemiology and pathophysiology of adulthood-onset thrombotic microangiopathy with severe ADAMTS13 deficiency (thrombotic thrombocytopenic purpura): a cross-sectional analysis of the French national registry for thrombotic microangiopathy. Lancet Haematol 2016;3(05): e237-e245

16 Scully M, Cataland S, Coppo P, et al; International Working Group for Thrombotic Thrombocytopenic Purpura. Consensus on the standardization of terminology in thrombotic thrombocytopenic purpura and related thrombotic microangiopathies. J Thromb Haemost 2017;15(02):312-322

17 Kremer Hovinga JA, Coppo P, Lämmle B, Moake JL, Miyata T, Vanhoorelbeke K. Thrombotic thrombocytopenic purpura. Nat Rev Dis Primers 2017;3:17020

18 Sadler JE. Pathophysiology of thrombotic thrombocytopenic purpura. Blood 2017;130(10):1181-1188

19 Scully M, Knöbl P, Kentouche K, et al. Recombinant ADAMTS-13: first-in-human pharmacokinetics and safety in congenital thrombotic thrombocytopenic purpura. Blood 2017;130(19): 2055-2063

20 Masias C, Cataland SR. Novel therapies in thrombotic thrombocytopenic purpura. Res Pract Thromb Haemost 2017;2(01): 19-26

21 Scully M, Cataland SR, Peyvandi F, et al; HERCULES Investigators. Caplacizumab treatment for acquired thrombotic thrombocytopenic purpura. N Engl J Med 2019;380(04):335-346

22 Kremer Hovinga JA, George JN. Hereditary thrombotic thrombocytopenic purpura. N Engl J Med 2019;381(17):1653-1662

23 Zheng L, Zhang D, Cao W, Song WC, Zheng XL. Synergistic effects of ADAMTS13 deficiency and complement activation in pathogenesis of thrombotic microangiopathy. Blood 2019;134(13):1095-1105

24 Roose E, Joly BS. Current and future perspectives on ADAMTS13 and thrombotic thrombocytopenic purpura. Hämostaseologie 2020;40:322-336

25 Roose E, Schelpe AS, Tellier E, et al. Open ADAMTS13, induced by antibodies, is a biomarker for subclinical immune-mediated thrombotic thrombocytopenic purpura. Blood 2020;136(03):353-361

26 Fujimura Y, Matsumoto M, Isonishi A, et al. Natural history of Upshaw-Schulman syndrome based on ADAMTS13 gene analysis in Japan. J Thromb Haemost 2011;9(Suppl 1):283-301

27 Fujimura Y, Lämmle B, Tanabe S, et al. Patent ductus arteriosus generates neonatal hemolytic jaundice with thrombocytopenia in Upshaw-Schulman syndrome. Blood Adv 2019;3(21):3191-3195

28 Tanabe S, Yagi H, Kimura T, et al. Two newborn-onset patients of Upshaw-Schulman syndrome with distinct subsequent clinical courses. Int J Hematol 2012;96(06):789-797 